\title{
Chapter
}

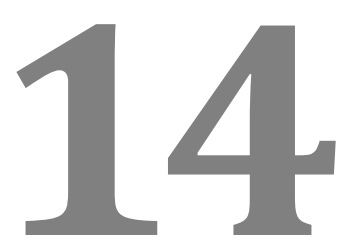

\section{BACTERIAL ADHESION ON POLYELECTROLYTE MULTILAYERS}

Klemen Bohinc $^{1 *}$ and Davor Kovačević ${ }^{2}$

${ }^{1}$ Faculty of Health Sciences, University of Ljubljana, Zdravstvena 5, 1000 Ljubljana, Slovenia

2 Division of Physical Chemistry, Department of Chemistry, Faculty of Science, University of Zagreb, Horvatovac 102a, 10000 Zagreb, Croatia 


\section{Contents}

14.1.INTRODUCTION .........................................................................................................................337

14.2. POLYELECTROLYTE MULTILAYERS.................................................................................338

14.3. APPLICATION OF POLYELECTROLYTE MULTILAYERS FOR STUDYING

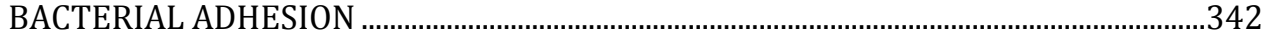

14.4. BACTERIAL ADHESION MEASUREMENTS......................................................................... 344

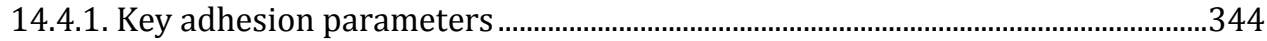

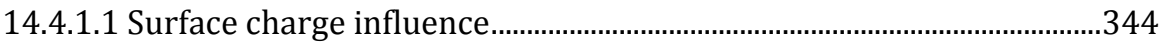

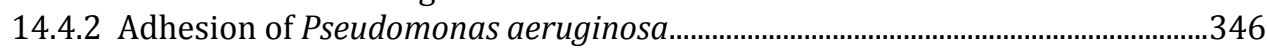

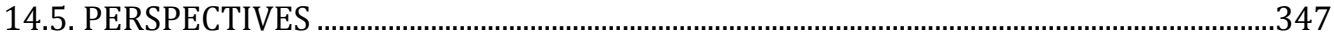

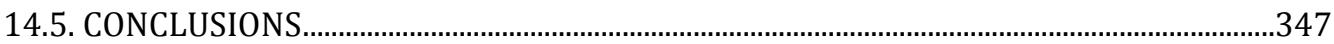

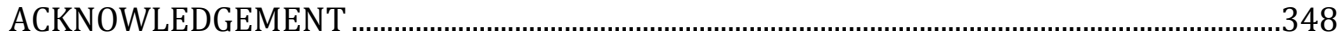

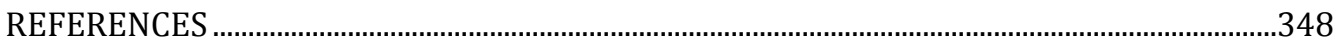




\subsection{INTRODUCTION}

In medicine, the interactions between microorganisms and material surfaces are of fundamental importance. In nature, bacteria can be present as planktonic cells, which are freely moving in a bulk solution [1]. The attractive interactions between microorganisms and surfaces can lead to bacterial adhesion, which is the first step in microbial colonization and biofilm formation. The biofilm has been defined as a complex, three-dimensional functional society of adherent microorganisms bound to, and growing at, an interface and encased by an extracellular polymeric matrix [2,3]. The adhesion process is the result of different physical and chemical processes. Various parameters, such as the properties of a microbial cell (cell surface hydrophobicity and charge, extracellular appendages, extracellular polymeric substances, signalling molecules), fluid (polarity, flow velocity, $\mathrm{pH}$, ionic strength, temperature, presence of salts, antimicrobials, nutrient availability), and surface chemistry (hydrophobicity, electric charge, surface roughness, etc.) can influence the bacterial adhesion [4].

Biofilms are clusters of microorganisms that are almost always found with healthcare-associated infections (involving medical devices, such as catheters, implants, pacemakers, and prosthetics). Vascular catheter-related bloodstream infections are the most serious infections [5]. Urinary catheters are made of tubular latex or silicone devices, and if inserted into the human body they may readily acquire biofilms on the inner or outer surfaces. With increasing time, the urinary catheter can become a place where microorganisms develop biofilms, resulting in urinary tract infections. Medical implants can be the location of device-related infections, which are difficult to eradicate because bacteria-causing infections live in well-developed biofilms [6]. The implants that can be compromised by biofilm associated infections are the following: heart valves, central venous catheters, ventricular assist devices, coronary stents, neurosurgical ventricular shunts, implantable neurological stimulators, fracture-fixation devices, arthro-prostheses, inflatable penile implants, breast implants, cochlear implants, intraocular lenses, and dental implants [7]. Biofilms on the previously mentioned medical devices are composed of grampositive or gram-negative bacteria or yeasts. Bacteria usually isolated from medical devices include the gram-positive Enterococcus faecalis, Staphylococcus aureus, Staphylococcus epidermidis, and Streptococcus viridans and the gram-negative Escherichia coli, Klebsiella pneumoniae, Proteus mirabilis, and Pseudomonas aeruginosa. These organisms usually originate from the skin of patients or workers in the health-care system [6]. Detachment of cells, production of endotoxin, increased resistance to the host immune system, and provision of a niche for the generation of resistant organisms can all result in biofilm production that may initiate an infection [8]. 
Biofilms also play an important role in antimicrobial-drug resistance. Bacteria present in biofilms are more resistant to antimicrobial agents than planktonic cells. The reason is the diminished rates of mass transport of antimicrobial molecules to the biofilm associated cells. On the other hand, the biofilm cells usually differ physiologically from planktonic cells [6].

Recently, many attempts have been made to develop coatings of implantable devices to reduce the bacterial adhesion and biofilm formation. A special, but very important, case of possible biomedical applications is the development of antibacterial polyelectrolyte multilayers (PEMs).

\subsection{POLYELECTROLYTE MULTILAYERS}

It is well-known that by mixing two aqueous solutions of positively and negatively charged polyelectrolytes that the aggregates form predominantly due to the electrostatic attraction between the oppositely charged chains. Such aggregates are generally known as polyelectrolyte complexes or interpolyelectrolyte complexes, although in early work, the terms complex flocculation and complex coacervation were also used. Stability of such complexes can be, in addition to predominant Coulombic forces, influenced by hydrogen bonds and hydrophobic interactions, etc. Structure and characteristics of such aggregates are determined by different factors, such as the nature of ionic groups, initial polyelectrolyte concentrations, $\mathrm{pH}$, ionic strength, temperature, and preparation procedure.

Among the first investigations in this field, the efforts of Bungenberg de Jong [9] and Kruyt [10] should be mentioned. They were following the formation and structure of polyelectrolyte complexes, at that time called complex coacervates. They investigated the influence of $\mathrm{pH}$ and molar mass of polycations and polyanions on the complex stability, as well as the condition of polyelectrolyte complex formation. Overbeek and Voorn [11] have tried to theoretically explain the experimental results obtained by Bungenberg de Jong and Kruyt. Using the Debye-Hückel theory, they determined the electrostatic energy and the entropy of mixing for two-component (polyelectrolytes and solvent) and three-component (polyelectrolytes, solvent, and inert electrolyte) systems. These early efforts in the field of polyelectrolyte complexes were followed by the Michaels' [12] studies of complexes formed by synthetic polyelectrolytes with high charge density. Later on, Dautzenberg $[13,14]$ investigated the structure and the characteristics of strong polyelectrolytes formed by sodium polystyrene sulfonate and poly(diallyldimethylammonium chloride) and their acrylamide copolymers. Using different experimental methods, he managed to determine the complex stoichiometry, the amount of bound sulfonate groups, the ratio of polycationic and polyanionic sites in the complex, as well as mass, size, and structural density of the complex. He 
showed that the presence of a small amount of salt during complex formation causes much lower values of the degree of aggregation. It is known that, especially for systems containing at least one weakly charged component, the type of supporting electrolyte and ionic strength influence the formation of aggregates. It was additionally shown by Dautzenberg and Karibyants [15] that complexes formed by strong polyanions (sodium polystyrene sulfonate) and strong polycations (poly(diallyldimethylammonium chloride)), as well as the complexes formed by weak polyanions (sodium polymethacrylate) and strong polycations (poly(diallyldimethylammonium chloride)) show in the presence of an inert electrolyte a lower degree of aggregation then in the absence of an inert electrolyte. The increase in ionic strength in such a system leads to secondary aggregation. Additionally, systems containing weak polyelectrolytes show a lower degree of aggregation in comparison with the systems containing strong polyelectrolytes. Numerous results in this field were obtained by Kabanov and his coworkers [16-20]. They investigated the formation, structure, and characteristics of different polyelectrolyte complexes obtained by mixing both strong and weak polyanions with strong polycations. The influence of ionic strength on the polyelectrolyte complex formation was thoroughly investigated by Pergushovet al. [21]. They used the fluorescence quenching method to explore the effect of different salts and the degree of polymerization on the nonstoichiometric complex formation.

Since 1991, when Decher introduced the layer-by-layer method of constructing PEMs on metal oxide surfaces [22], interest in the process of formation of such layered structures has been continuously growing.
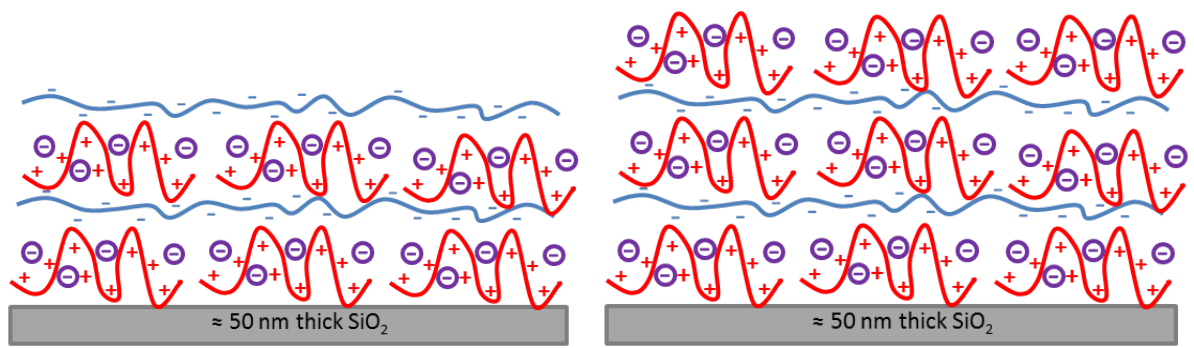

Figure 1. Scheme of PEMs having a negative (left) and a positive (right) terminating layer formed on a silica surface

Various experimental methods [23,24] applied to obtain a better insight into the process has helped increase the understanding of the multilayer formation process. The ease of formation of multilayers motivated scientists to extend the type of constituents incorporated into such nanocomposites by including proteins [25], dendrimers [26], and even DNA [27,28]. 
As stated above, the layer-by-layer deposition method to prepare multilayers of polyelectrolytes of alternating charge can be followed by various experimental methods. Very interesting results were obtained by stagnation point optical reflectometry [29,30], ellipsometry [25], quartz crystal microbalance [31], and optical waveguide lightmode spectroscopy [32]. Here, we will concentrate on the cases where different effects have been followed in situ by means of optical reflectometry experiments [29,30,33,34]. It turns out that in solutions containing both polyelectrolyte and appropriate salts up to a certain concentration, the regular build-up of multilayers is modified and becomes an adsorption/redissolution process [28]. This was explained by taking into account (i) that during the regular multilayer formation process the macromolecules cannot equilibrate, (ii) that the added salt plasticizes the multilayer to a state where the molecules are sufficiently mobile to enable them to equilibrate between the layer and the surrounding solution, and (iii) that the presence of excess polyelectrolyte brings the system to a one-phase region of the polyelectrolyte complex phase diagram, implying that polyelectrolyte complexes must dissolve under these conditions.

Additionally, the influence of different salts (phosphates, chlorides, and nitrates) and polyelectrolyte molecular weight on the formation and erosion of multilayers on silica surfaces was also investigated by means of the optical reflectometry method [30]. In all of these experiments, the anionic polyelectrolyte was poly(acrylic acid). On the other side, three different cationic polyelectrolytes were used: poly(dimethylaminoethyl)methacrylate, poly(allylamine hydrochloride), and poly(2-vinyl- $N$-methylpyridinium iodide). It has been shown that at very low ionic strength $(1 \mathrm{mM})$ regular build-up of multilayers is observed independent of the salt used. However, at higher ionic strength, dissolution also takes place, and the critical "glass transition ionic strength" needed for the multilayer to be dissolved depends on the salt used, as well as on the polycation/polyanion pair studied.

The application of optical reflectometry for the characterization of multilayers obtained by exposing a suitable substrate to solutions of a cationic homopolymer and an oppositely charged protein (which could also be of interest for studying bacterial adhesion) was also performed [33]. In these experiments, the negatively charged component bovine serum albumin (BSA) was used. As the cationic homopolymer, a weak polyelectrolyte poly(dimethylaminoethyl)methacrylate (PAMA) or a strong polyelectrolyte poly(2-vinyl- $N$-methylpyridinium iodide) $\left(\mathrm{PVP}^{+}\right)$were used. As the solid substrate, we used silica in the form of silicon wafers carrying an oxide layer, and as the supporting electrolyte we used potassium chloride or phosphate buffer. The influence of ionic strength, $\mathrm{pH}$, type of cationic homopolymer (strong or weak), and protein concentration were investigated, and it was shown that stagnation point optical reflectometry can be a useful method for characterization of the formation of multilayers containing polyelectrolyte and protein layers. The method of stagnation point optical reflectometry was also 
applied for examining the adsorption of BSA on the previously formed poly(allylamine hydrochloride)/poly(sodium 4-styrenesulphonate) (PAH/PSS) multilayer, with PAH being a terminal layer [34]. The solid substrate was silica in the form of silicon wafers carrying an oxide layer. In order to interpret the adsorption of BSA, the build-up mechanism of the PAH/PSS multilayers was examined, with special emphasis on the effect of electrolyte concentration, $\mathrm{pH}$ of solution, and the anchoring (precursor) layer on that process. Additionally, the effect of BSA concentration and the anchoring layer on BSA adsorption was investigated. It was shown that in all investigated systems, the adsorption of BSA depends on the conditions under which the multilayer was formed (ionic strength, $\mathrm{pH}$, and presence of an anchoring layer), as well as on BSA concentration. It follows that the adsorption of BSA could be controlled not only by choosing suitable BSA concentration, but also by modifying the preformed multilayer.

Since layer-by-layer structures (e.g. PEMs and protein-PEMs) play a very important role in surface modification processes, it was intriguing to check if other experimental methods would confirm the results and conclusions obtained by stagnation point optical reflectometry. Therefore, electrokinetic measurements were also applied for the investigation of PAH/PSS multilayer formation as a function of $\mathrm{pH}$, with $\mathrm{PAH}$ being a terminal layer [35]. The instrument uses electrophoretic light scattering and the Laser Doppler Velocimetry method for determination of particle velocity, and from this the electrokinetic zeta potential can be determined. The electrokinetic potential was calculated from mobility values using the Smoluchowski equation. Additionally, the effect of supporting electrolyte $(\mathrm{KCl})$ concentration on multilayer formation was tested. Silica particles were used as the solid substrate. The adsorption of BSA on the previously formed multilayer was examined as a function of $\mathrm{pH}$ and BSA concentration. The experiments were performed at three different ionic strength values as a function of $\mathrm{pH}$, and it was confirmed that the electrokinetic measurements were suitable for monitoring the formation of various multilayers. In all investigated systems, the process of multilayer formation was found to depend on conditions (ionic strength and $\mathrm{pH}$ ) under which the multilayer was formed. Moreover, BSA concentration also played a significant role in the adsorption on the previously formed multilayer.

- All the above mentioned results (both the results that concern polyelectrolyte complexes as well as the ones that concern PEMs) were shown to depend on so called ionic conditions. That means that the formation processes are very sensible to the concentration of added supporting electrolyte, but also to the electrolyte type. In order to obtain a deeper insight into effects occurring when electrolyte solution is added to solution of a strong polyelectrolyte, the microcalorimetric and potentiometric titrations of poly(sodium 4-styrenesulfonate) $\left(\mathrm{Na}^{+} \mathrm{PSS}^{-}\right)$solution with different alkali, earth-alkali, and 
tetraalkylammonium nitrate, perchlorate, and chloride solutions were performed [36]. From the calorimetric titrations, the differences in sign and magnitude of enthalpy change upon addition of various electrolytes were observed depending on the salt used. Potentiometric titrations using a sodium ion-selective electrode have revealed that addition of electrolyte is accompanied by the increase in sodium activity until a certain critical value is reached, which seems to be the consequence of counterion substitution on the polyelectrolyte chain. In the case of the addition of lithium and sodium salts, the experimental results for the change in enthalpy of mixing $(\Delta H)$ can be qualitatively correctly explained by the Poisson-Boltzmann and Monte Carlo calculations based on the continuum solvent models. This is not the case for the mixtures with $\mathrm{KNO}_{3}, \mathrm{RbNO}_{3}$, and $\mathrm{CsNO}_{3}$ salts. The results suggest that the ion-specific effects, associated with the changes in water structure, have to be taken into account when thermodynamic properties of polyelectrolytes in solution are concerned. The calorimetric results imply that the enthalpically observed cation specificity for binding to the poly(styrenesulfonate) group could be correlated with the corresponding cation hydration enthalpies. The counterion substitution of sodium with divalent cations was found to be endothermic, which is in qualitative agreement with the electrostatic theory.

\subsection{APPLICATION OF POLYELECTROLYTE MULTILAYERS FOR STUDYING BACTERIAL ADHESION}

The adsorption of biological or biomimetic structures onto certain synthetic materials (in this case PEMs) could enable additional progress in the field of biosensing surfaces, tissue engineering, and drug delivery. PEMs formed by alternate adsorption of positively and negatively charged polyelectrolytes are promising coatings onto which biological molecules (e.g. proteins) could be adsorbed. In the literature [37-39], several examples of investigation of adsorption of different proteins on previously formed multilayers using different experimental methods can be found. Müller and coworkers [37] examined the sorption of human serum albumin (HSA) on poly(ethyleneimine)/poly(acrylic acid) multilayers using attenuated total reflection Fourier transform infrared (ATR FTIR) spectroscopy, while Gergelly etal. [38] analysed adsorption of the same protein on poly(Llysine)/poly(glutamic acid) multilayer by Optical Waveguide Light-Mode Spectroscopy (OWLS) and atomic force microscopy (AFM). The secondary structure of BSA adsorbed onto PAH/PSS multilayers was also investigated by 
Schaaf and coworkers [39], and it was observed that PAH as the terminal layer has practically no effect on aggregation of BSA.

A special and very promising case is the possible application of PEMs as antibacterial coatings [40-44]. Many investigated PEMs promote or disrupt bacterial biofilm formation simply because of their high surface charge density. If the protruding chains bear an opposite charge than the bacteria, then these become attached to the PEM surfaces. In the case of their like charge, the adhesion is hindered. Zan and Shu [40] proposed a scheme for the procedure for fabricating PEMs containing silver ions or silver nanoparticles, which could be used as effective antibacterial coatings. On the other hand, Wong and coworkers showed that protein adsorption is drastically lowered on microbicidal hydrophobic/hydrophilic PEMs [41].

In addition to charge, factors that could affect the intensity of bacterial adhesion include material surface roughness, charge, degree of hydrophobicity, Lewis acid-base character, and hydrogen-bonding capacity [45-48]. Environmental factors, including $\mathrm{pH}$, temperature, nutrient composition, and population characteristics may enhance the adhesion and biofilm maturation [48].

Taking into account that PEM-modified surfaces could exhibit significantly improved bacterial anti-adhesive properties, it is important to relate the conditions (polyelectrolyte concentration, salt concentration and type) at which the PEMs are formed, the number of polyelectrolyte layers, and the type of terminating layer with the corresponding adhesion of bacteria. 


\subsection{BACTERIAL ADHESION MEASUREMENTS}

\subsubsection{Key adhesion parameters}

As stated above, to study bacterial adhesion rate we need to first characterize the material (in this case PEM) and the bacterial surfaces to determine the key parameters in the adhesion process. For example, one of these parameters is surface roughness, which could be measured with AFM or profilometry. Surface hydrophobicity can be obtained from contact angle measurements, while the surface charge can be determined from electrophoretic or streaming potential measurements. On the other hand, many methods for adhered bacteria counting have been published, including direct counting methods, such as scanning electron microscopy (SEM), and indirect counting methods, such as colony forming units (CFU), plate count, and staining methods $[49,50]$.

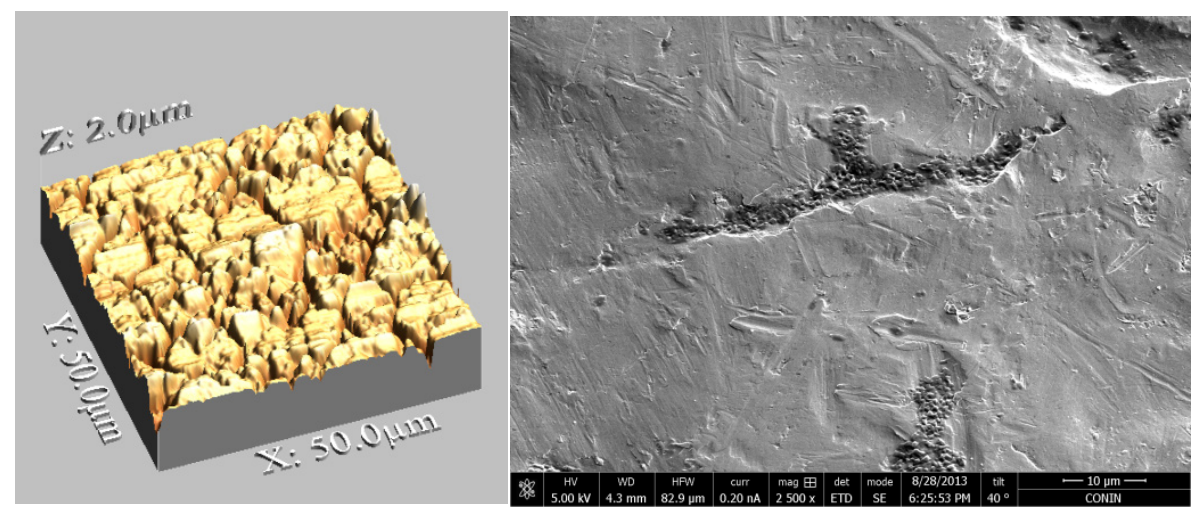

Figure 2. Left: AFM picture of metal surface AISI 304 3C. The root mean square (RMS) roughness is $160.5 \mathrm{~nm}$. Right: SEM picture of adhered bacteria on electropolished surfaces after $24 \mathrm{~h}$.

\subsubsection{Surface charge influence}

As stated above, one of the key factors that influences the intensity of bacterial adhesion is the charge of the surface. For that purpose, zeta-potential measurements can be used. In our earlier study [51], we applied zeta-potential measurements for studying the properties of the PEMs formed from poly(allylamine hydrochloride), $\mathrm{PAH}$, and sodium poly(4-styrenesulfonate), PSS. These synthetic polyelectrolytes have been widely used in the process of PEM formation, and we also used them extensively in our investigations of polyelectrolyte complexes [52]. The example of the change in surface charge of PEMs (i.e. the change in zeta-potentials) is presented in Figure 3 for the formation of PAH/PSS multilayers. 


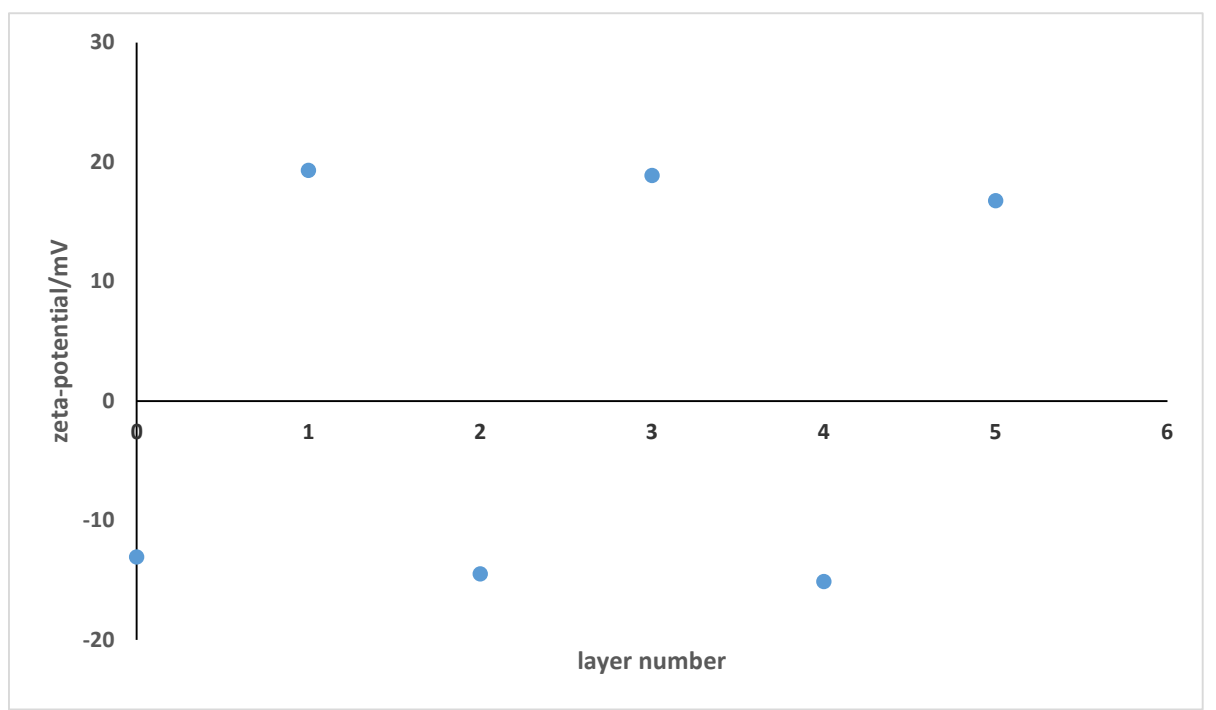

Figure 3. The dependence of the zeta-potential on the number of oppositely charged polyelectrolyte layers in the PAH/PSS system in the presence of sodium perchlorate; $c_{\mathrm{m}}(\mathrm{PAH})=c_{\mathrm{m}}(\mathrm{PSS})=0.001 \mathrm{~mol} \mathrm{dm}^{-3}, c\left(\mathrm{NaClO}_{4}\right)=0.001 \mathrm{~mol} \mathrm{dm}^{-3}, t=25^{\circ} \mathrm{C}$.

We showed [51] that polyelectrolyte concentration, as well as supporting electrolyte (added salt) concentration, significantly influence the multilayer build-up. Therefore, in the process of PEM formation, special attention should be given to controlling these experimental parameters. Of course, other experimental conditions which are known to influence PEM build-up, such as $\mathrm{pH}$, polyelectrolyte type, added supporting electrolyte type, molecular weight, and temperature should be also taken into account.

The importance of charge for bacterial adhesion was also shown by Lienkamp and coworkers [53]. They prepared multilayers formed from poly(acrylic acid) (PAA) and either the hydrophobic butyl synthetic mimics of antimicrobial peptides (SMAMP) or the hydrophilic diamine SMAMP with a poly(ethylenimine) anchoring layer, and found that the positive charge of PEMs with SMAMP as the terminating layer is low, since a significant part is consumed to maintain layer stability. This leads to reduced antimicrobial activity. The suppression of biofilm formation can also be achieved by introducing strongly hydrophilic groups at the surface of PEMs. However, the prevention of bacterial adhesion cannot result in their complete elimination. This can be achieved once stable bacteria attachment is achieved. 


\subsubsection{Influence of other surface parameters}

As stated above, other key parameters, such as surface hydrophobicity and surface roughness, should be examined. In the case of PEMs formed on oxidized silicon wafers prior to adhesion of bacteria, the obtained hydrophobicity (i.e. contact angle) was similar on PEMs terminating with a polycation and with a polyanion layer. In the first case, the contact angle was $48.9^{\circ} \pm 2.5^{\circ}$ and in the latter one the contact angle was $46.9^{\circ} \pm 5.0^{\circ}$.

The results of roughness measurements obtained by means of atomic force microscopy did not differ much in the case of the two types of multilayers. In the case of the positive surface (polycation terminating multilayer), roughness was determined to be $0.017 \mu \mathrm{m} \pm 0.004 \mu \mathrm{m}$, and in the case of the negative surface (polyanion terminating multilayer), roughness was $0.019 \mu \mathrm{m} \pm 0.006 \mu \mathrm{m}$.

Table 1. PEMs with terminating layers bearing positive or negative charge investigated in terms of the contact angle value and roughness. Adapted from [51].

\begin{tabular}{ccc}
\hline & $\begin{array}{c}\text { Positively charged } \\
\text { polyelectrolyte PAH } \\
\text { as terminating layer } \\
\text { (5 layers) }\end{array}$ & $\begin{array}{c}\text { Negatively charged } \\
\text { polyelectrolyte PSS as } \\
\text { terminating layer } \\
\text { (6 layers) }\end{array}$ \\
\hline Contact angle & $48.9^{\circ} \pm 2.5^{\circ}$ & $46.9^{\circ} \pm 5.0^{\circ}$ \\
\hline Roughness & $0.017 \mu \mathrm{m} \pm 0.004 \mu \mathrm{m}$ & $0.019 \mu \mathrm{m} \pm 0.006 \mu \mathrm{m}$ \\
\hline
\end{tabular}

\subsubsection{Adhesion of Pseudomonas aeruginosa}

For examining the adhesion of bacteria, $P$. aeruginosa is very often used. $P$. aeruginosa is a common pathogenic bacterium with a possible multidrug resistance by mutation and could be responsible for various possible postoperative infections. Recently, Dekany and coworkers showed [54] that clinically relevant pathogen strains (for example, P. aeruginosa) could be inactivated by the photocatalytically active titanium dioxide functionalized with silver nanoparticles and immobilized in polyacrylate-based nanohybrid thin film [55]. In our study [51], we showed that the fraction of the multilayer surface covered with P. aeruginosa was $20.4 \% \pm 4.8 \%$, with PAH as the terminating layer, and $9.0 \% \pm 3.1 \%$, with PSS as terminating layer. These results allowed the assumption that differences in bacterial adhesion capability between the systems with oppositely charged terminating layers should be the result of electrostatic interactions. That is in accordance with predictions since the bacteria cell walls possess negative charges. Bohinc et al. [46] measured the zeta potential of $P$. aeruginosa for two phosphate buffer solutions at two ionic strengths, $1 \mathrm{mmoll}^{-1}$ and $100 \mathrm{mmoll}^{-1}$, and the negative 
zeta potentials were obtained as follows: $-16.92 \mathrm{mV} \pm 2.42 \mathrm{mV}$ for $1 \mathrm{mmoll}^{-1}$ and $-7.85 \mathrm{mV} \pm 12.8 \mathrm{mV}$ for $100 \mathrm{mmoll}^{-1}$.
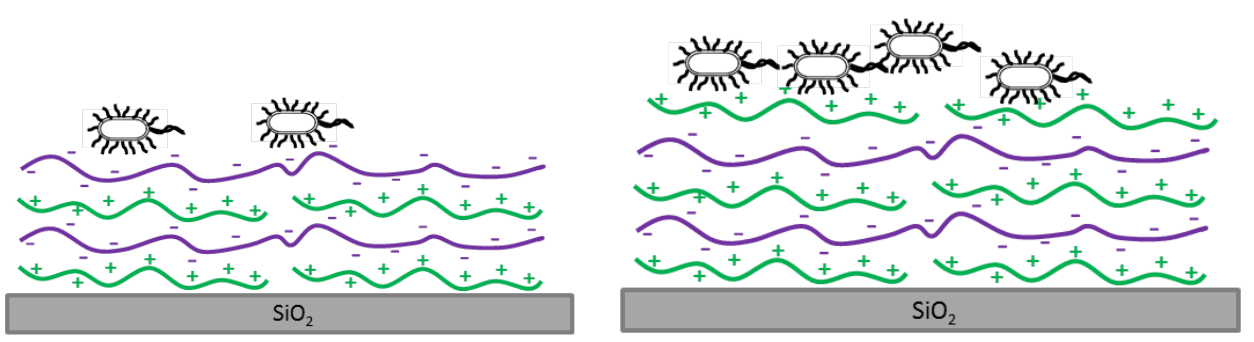

Figure 4. Schematic presentation of the adhesion of bacteria on PEMs with negative (left) and positive (right) terminating layers [51]

\subsection{PERSPECTIVES}

In recent years, significant progress in the design of surface coatings has been made [43]. Generally, there are two possibilities for preventing bacterial adhesion to surfaces and subsequent bacterial infections, which are physical and pharmacological. PEMs can be used for both attempts. They can be applied as antifouling coatings in which antibacterial substances, such as antibiotics, can be incorporated. Antibacterial substances are then slowly released.

In the existing antimicrobial implant coatings, drug dosage and release rate cannot be easily tuned. Local sustained delivery is still difficult to achieve. For these problems, specific PEMs [51] can improve the efficiency and tunability of the drug dosage [56].

In many cases, the initial contamination of medical devices is mostly caused by a small number of microorganisms. They are often transferred to the device via the patient's or healthcare workers' skin, contaminated water, or other external environmental sources. Infections in medicine cause a huge financial burden on healthcare services. On the other hand, infections are responsible for the patient's morbidity and mortality. To solve the mentioned problems, PEMs on implantable devices are especially attractive.

\subsection{CONCLUSIONS}

In this chapter, we considered the bacterial adhesion on material surfaces coated with PEMs. Generally, bacterial adhesion can be controlled by different material and bacterial surface properties, such as surface roughness, surface 
charge, hydrophobicity, and specific surface structure. It is important to state here that the combination of surface characterization with microbial testing leads to the better understanding of the bacteria-surface interactions. Regarding surfaces coated with PEMs, it seems that the main factor for bacterial adhesion is the surface charge. In terms of PEM formation, adjusting salt type and concentration, as well as the appropriate terminating polyelectrolyte layer, can lead to the formation of multilayer systems with optimized antibacterial properties.

\section{ACKNOWLEDGEMENT}

The authors are thankful to the Croatian Science Foundation (project IP-201409-6972) and to the COST Action CA15216 European Network of Bioadhesion Expertise: Fundamental Knowledge to Inspire Advanced Bonding Technologies for the support. The authors thank Dr. Goran Dražić for Figure 2.

\section{REFERENCES}

1. T.R. Garrett, M. Bhakoo, Z. Zhang, Prog. Nat. Sci.18 (2008) 1049-1056.

2. D. Mack, A.P. Davies, L.G. Harris, H. Rohde M.A. Horstkotte, J.K. Knobloch, Anal. Bioanal. Chem. 387 (2007) 399-408.

3. T. Kawarai, S. Furukawa, N. Narisawa, C. Hagiwara, H. Ogihara, M. Yamasaki, J. Biosci. Bioeng. 107 (2009) 630-635.

4. K. Bohinc, M. Oder, R. Fink, K. Godič Torkar, G. Dražić, P. Raspor, Factors Affecting Microbial Adhesion in Pharmaceutical, Biomedical, and Dental Fields, K.L. Mittal, F.M. Etzler (Eds.), 2017, pp. 167-182.

5. M.A. Ryder, Topics Adv. Practice Nurs. e-J. 5 (2005) .

6. R. Donlan, Emerg. Infect. Dis. 7(2001) 277-281.

7. J.W. Costerton, L. Montanaro, C.R. Arciola, Int. J. Arti. Organs. 28 (2005) 10621068.

8. P.V. Vlastarakos, T.P. Nikolopoulos, P. Maragoudakis, A. Tzagaroulakis, E. Ferekidis, Laryngoscope 117 (2007) 668-673.

9. H.G. Bungenberg de Jong, in: H.R. Kruyt (Ed.), Colloid Science, vol. II, Elsevier Publishing Company, Amsterdam, 1949, pp. 335-384.

10. H.R. Kruyt, Colloid Science, vol. II, Elsevier Publishing Company, Amsterdam, 1949.

11. J.T.G. Overbeek, M.J.J. Voorn, J. Cell. Comp. Physiol. 49 (1957) 7-26.

12. A.S. Michaels, Ind. Eng. Chem. 57 (1965) 32-40.

13. H. Dautzenberg, in: T. Radeva (Ed.), Physical Chemistry of Polyelectrolytes, Marcel Dekker Inc., New York, 2001, pp. 743-792.

14. H. Dautzenberg, Macromolecules 30 (1997) 7810-7815.

15. H. Dautzenberg, N. Karibyants, Macromol. Chem. Phys. 200 (1999) 118-125. 
16. V.A. Kabanov, Fundamentals of Polyelectrolyte Complexes in Solution and the Bulk, in: G. Decher, J. Schlenoff (Eds.), Multilayer Thin Films, Wiley-VCH, 2003, pp. 47-86.

17. V.A. Kabanov, Soviet. Sci. Rev., Ser. B, Chem. Rev. 4 (1982) 207-282.

18. D.V. Pergushov, V.A. Izumrudov, A.B. Zezin, V.A. Kabanov, Polym. Sci. 35 (1993) 844-849.

19. D.V. Pergushov, V.A. Izumrudov, A.B. Zezin, V.A. Kabanov,. Polym. Sci. 37 (1995) 1081-1087.

20. D.V. Pergushov, E.V. Remizova, J. Feldthusen, A.B. Zezin, A.H.E. Müller, V.A. Kabanov, J. Phys. Chem. B 107 (2003) 8093-8096.

21. D.V. Pergushov, H.-M. Buchhamer, K. Lunkwitz, Colloid. Polym. Sci. 277 (1999) 101-107.

22. G. Decher, J.D. Hong, Macromol. Chem. Macromol. Symp. 46 (1991) 321-327.

23. R.V. Klitzing, Phys. Chem. Chem. Phys. 8 (2006) 5012-5033.

24. M. Schönhoff, Curr. Opinion Colloid Interface Sci. 8 (2003) 86-95.

25. P. Schwinte, J.C. Voegel, C. Picart, Y. Haikel, P. Schaaf, B. Szalontai, J. Phys. Chem. B 105 (2001) 11906-11916.

26. B.Y. Kim, M.L. Bruening, Langmuir 19 (2003) 94-99.

27. J.T. Zhang, L.S. Chua, D.M. Lynn, Langmuir 20 (2004) 8015-8021.

28. B. Sun, D.M. Lynn, J. Controlled Release 148 (2010) 91-100.

29. D. Kovačević, S. van der Burgh, A. de Keizer, M.A. Cohen Stuart, Langmuir 18 (2002) 5607-5612.

30. D. Kovačević, S. van der Burgh, A. de Keizer, M.A. Cohen Stuart, J. Phys. Chem. B 107 (2003) 7998-8002.

31. L. Wagberg, G. Pettersson, S. Notley, J. Colloid Interface Sci. 274 (2004) 480488.

32. C. Picart, Ph. Lavalle, P. Hubert, F.J.G. Cuisinier, G. Decher, P. Schaaf, J.C. Voegel, Langmuir 17 (2001) 7414-7424.

33. D. Kovačević, S. Glavanović, N. Peran, Colloids Surf. A 277 (2006) 177-182.

34. E. Primorac, I. Đapić, N. Štrbe, D. Kovačević, Colloids Surf. B 76 (2010) 305310.

35. I. Đapić, D. Kovačević, Croat. Chem. Acta 84 (2011) 185-191.

36. J. Požar, K. Bohinc, V. Vlachy, D. Kovačević, Phys. Chem. Chem. Phys. 13 (2011) 15610-15618.

37. M. Müller, B. Kessler, W. Ouyang, Z. Phys. Chem. 221 (2007) 127-138.

38. C. Gergely, S. Bahi, B. Szalontai, H. Flores, P. Schaaf, J.C. Voegel, F.J.G. Cuisinier, Langmuir 20 (2004) 5575-5582.

39. P. Schwinte, V. Ball, B. Szalontai, Y. Haikel, J.C. Voegel, P. Schaaf, Biomacromolecules 3 (2002) 1135-1143.

40. X. Zan, Z. Su, Thin Solid Films 518 (2010) 5478-5482.

41. S.Y. Wong, L. Han, K. Timachova, J. Veselinovic, M.N. Hyder, C. Ortiz, A.M. Klibanov, P.T. Hammond, Biomacromolecules 13 (2012) 719-726.

42. L. Tang, W. Gu, P. Yi, J.L. Bitter, J.Y. Hong, D.H. Fairbrother, K.L. Chen, J. Membr. Sci. 446 (2013) 201-211.

43. J.A. Lichter, M.F. Rubner, Langmuir 25 (2009) 7686-7694.

44. T. Kruk, K. Szczepanowicz, D. Kręgiel, L. Szyk-Warszyńska, P. Warszyński, Colloids Surf. B: Biointerfaces 137 (2016) 158-166.

45. R. Bos, H.C. van der Mei, H.J. Busscher, FEMS Microbiol. Rev. 23 (1999) 179230. 
46. K. Bohinc, G. Dražić, R. Fink, M. Oder, M. Jevšnik, D. Nipič, K. Godič Torkar, P. Raspor, Int. J. Adhes. Adhes. 50 (2014) 265-272.

47. K. Bohinc, G. Dražić, A. Abram, M. Jevšnik, B. Jeršek, D. Nipič, M. Kurinčič, P. Raspor, Int. J. Adhes. Adhes. 68 (2016) 39-46.

48. P.J. Herrald, E.A. Zootolla, J. Food Sci. 51 (1988) 445-448.

49. E.M. Jaryszak, E.M. Sampson, P.J. Antonelli, Curr. Opin. Otolaryngol. Head Neck Surg. 30 (2009) 367-370.

50. Y.H. An, R.J. Friedman, J. Microbiol. Methods 30 (1997) 141-152.

51. D. Kovačević, R. Pratnekar, K. Godič Torkar, J. Salopek, G. Dražić, A. Abram, K. Bohinc, Polymers 8 (2016) 345:1-345:12.

52. J. Požar, D. Kovačević, Soft. Matter. 10 (2014) 6530-6545.

53. F. Dorner, A. Malek-Luz, J.S. Saar, S. Bonaus, A. Al-Ahmad, K. Lienkamp, Macromol. Chem. Phys. 217 (2016) 2154-2164.

54. S.P. Tallósy, L. Janovák, E. Nagy, A. Deák, Á. Juhász, E. Csapó, N. Buzás, I. Dékány, Appl. Surf. Sci. 371 (2016) 139-150.

55. L. Janovák, A. Deák, S.P. Tallosy, D. Sebok E. Csapo, K. Bohinc, A. Abram, I. Palinko, I. Dékány, Surf. Coat. Technol. 326 (2017) 316-326.

56. V.B. Teif, K. Bohinc, Progr. Biophys. Mol. Biol. 105 (2011) 208-222.

(C)2018 by the authors; licensee IAPC, Zagreb, Croatia. This chapter is an open-access publication distributed under the terms and conditions of the Creative Commons Attribution license (http://creativecommons.org/licenses/by/3.0/) (cc) BY 